\title{
Genetic Variation in the $\kappa$-Casein Gene (CSN3) of Chinese Yak (Bos grunniens) and Phylogenetic Analysis of CSN3 Sequences in the Genus Bos
}

\author{
E.-M. Prinzenberg, ${ }^{\star 1}$ H. Jianlin, $† \ddagger$ and G. Erhardt* \\ *Institute of Animal Breeding and Genetics, Justus-Liebig-University, 35390 Giessen, Germany \\ †College of Animal Science, Gansu Agricultural University, Lanzhou 730070, Gansu, China

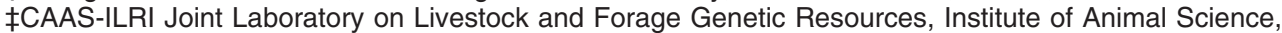 \\ Chinese Academy of Agricultural Sciences (CAAS), Beijing 100094, China
}

\begin{abstract}
Variants of $\kappa$-casein (CSN3) have been extensively studied in cattle and 13 alleles have been identified at the protein and DNA levels to date. Evolution of some of these alleles and a possible common ancestor remain unclear. Polymerase chain reaction-single strand conformation polymorphism (PCR-SSCP) analysis of CSN3 exon IV in domesticated yak revealed a 2-allele polymorphism showing migration patterns different from known cattle variants. The PCR products of both yak CSN3 SSCP alleles were sequenced. All yak had nucleotide sequences coding for Thr in AA position 136 (identical to bovine $C S N 3 * A$ ) and Ala in position 148 (identical to bovine $C S N 3 * B$ ). The sequencing results were confirmed by PCR-RFLP analysis using HindIII and TaqI. A 12-bp insertion in the coding region, representing a repeated nucleotide and AA motif, was found in 1 yak allele. The duplication corresponds to the codons for AA 147 to 150 (Glu-Ala-Ser-Pro) or 148 to 151 (Ala-Ser-ProGlu), which are repeated identically. In 21 yak samples genotyped by PCR-SSCP analysis, frequencies for the insertion variant and the short variant were about 68 and $32 \%$, respectively. The loss of the insertion may have led to the ancestral CSN3 allele from which all currently known variants of CSN3 in the genus Bos evolved. This is the first report of polymorphisms in the yak CSN3 gene and may be helpful for future studies on genetic variation within and between yak populations or on associated traits.
\end{abstract}

Key words: yak, $\kappa$-casein, CSN3 gene, polymorphism

\section{INTRODUCTION}

Variants of $\kappa$-casein (CSN3) have been extensively studied for decades. Altogether, 13 alleles have been

\footnotetext{
Received October 2, 2007.

Accepted December 8, 2007.

${ }^{1}$ Corresponding author: eva-maria.prinzenberg@agrar. uni-giessen.de
}

identified in the Bos genus using either electrophoretic separation of protein variants, protein and DNA sequence analyses, or various DNA mutation detection techniques. These are summarized, including conflicting allele names, by Formaggioni et al. (1999) and Farrell et al. (2004). Up to 9 alleles (A, B, C, E, F, G, H, I, and $A^{I}$ ) have been simultaneously discriminated in $B o s$ taurus and Bos indicus by PCR-single strand conformation polymorphism (SSCP) analysis so far (Prinzenberg et al., 1999). Evolution of these alleles remains unclear particularly for $C S N 3^{*} A$ and $C S N 3^{*} B$, with 2 mutations discriminating these alleles. A possible common ancestor and the presence of an intermediate ancestral protein were postulated earlier (Mercier et al., 1976; Gorodetskii and Kaledin, 1987). Udina et al. (1995) identified a $C S N 3$ variant named $C S N 3{ }^{*} G$ by PCRRFLP analysis in European bison (Bison bonasus), which corresponds to bovine $C S N 3 * A$ in codon 136 (Thr) and to $C S N 3 * B$ in codon 148 (Ala), thus representing such an intermediate protein. Based on microsatellite data, Ritz et al. (2000) proposed that Bos taurus, Bos indicus, and yak should be considered as subgenera of the Bos genus, whereas Bison clearly forms a separate genus. Sulimova et al. (1996) identified in yak a CSN3 exon IV sequence similar to that found in Bison with an additional mutation changing the stop codon from TAA to TGA in yak. Fan et al. (2000) also reported a unique yak CSN3 exon IV sequence (published in GenBank with accession no. AF194989). A $\kappa$-casein "X" protein variant of Nepalese yak (Kawamoto et al., 1992) was never characterized at either the AA or nucleotide sequence level.

In contrast to cattle, $\beta$-casein and $\kappa$-casein were found to be monomorphic in yak milk (Mao et al., 2004). Some studies about genetic variation of milk proteins within and between yak populations have been performed (Xuebin and Jianlin, 2000; Yucai et al., 2000). They also suggest that genetic variation is very low at the protein level and mainly occurs because of cattle introgression through hybridization. The aim of this study 
was to identify and characterize the genetic variation in Chinese yak CSN3 at the DNA sequence level and to establish the phylogenetic relationship of CSN3 variants in the Bos genus.

\section{MATERIALS AND METHODS}

\section{DNA Samples}

Blood samples of 15 male and 6 female yak (Bos grunniens / Poëphagus) were collected from 4 domesticated yak herds (4 to 6 samples per herd) in Luqu county of Gannan prefecture, Gansu Province, China, by a member of the Gansu Agricultural University staff in 1998. The DNA was extracted using essentially the high salt protocol of Montgomery and Sise (1991).

\section{PCR-SSCP Analysis}

The coding region of exon IV of the CSN3 gene was PCR amplified as described by Prinzenberg et al. (1999) using primers ExIV\#1 (5' AGA AAT AAT ACC ATT CTG CAT $\left.3^{\prime}\right)$ and 551'2 (5' GTT GAA TTC TTT GAT GTC TCC TTA GAG T $3^{\prime}$ ). The fragment comprised positions 4,892 to 5,442 of the CSN3 gene (accession number X14908; Alexander et al., 1988). Aliquots of $4 \mu \mathrm{L}$ of each PCR reaction were mixed with $6 \mu \mathrm{L}$ of denaturing loading dye (containing 95\% wt/vol formamide), heated at $92^{\circ} \mathrm{C}$ for $2 \mathrm{~min}$, and chilled on ice. Subsequently, $4 \mu \mathrm{L}$ of the mixture was loaded on $8.5 \%$ polyacrylamide gels (acrylamide:bisacrylamide ratio of 37:1) containing $0.53 \%$ glycerol. Fragments were separated for $18 \mathrm{~h}$ at $340 \mathrm{~V}$ and $5^{\circ} \mathrm{C}$ constant temperature. For silver staining, the protocol of Bassam et al. (1991) was used with a modified stop solution $(0.04 \mathrm{M}$ NaEDTA at $10^{\circ} \mathrm{C}$ ). Alleles were manually scored on a white, translucent screen using cattle samples with known genotypes, including the alleles $C S N 3^{*} A, B, C$, $E, G, H$, and $I$ as reference samples (Prinzenberg et al., 1999).

\section{PCR-RFLP Analyses}

For restriction enzyme digestion, 10 to $15 \mu \mathrm{L}$ of PCR product of each SSCP type was incubated with $5 \mathrm{U}$ of HindIII, TaqI, and MspI (MBI Fermentas, St. Leon Roth, Germany) following the manufacturer's guidelines. The fragments were separated by electrophoresis in $1.2 \%$ agarose gels and visualized by ethidium bromide staining and UV-fluorescence. Fragments were scored manually by comparison with a 100-bp ladder.

\section{Cloning and Sequencing}

The PCR products for cloning and sequencing were prepared from 8 yak (2 of type 11, 3 of type 12, and 3 of type 22) using the same primers and conditions as for the PCR-SSCP and PCR-RFLP analyses except for the use of Pwo polymerase with the buffer supplied by the manufacturer (AGS, Heidelberg, Germany). Bluntended PCR products were gel purified and cloned into $p C R$-Blunt using the Zero Blunt PCR Cloning kit (Invitrogen, Groningen, the Netherlands). Positive clones were selected by colony PCR and subsequent SSCP analysis of the respective PCR products. Clones with migration patterns different from those of PCR products derived from the genomic DNA were discarded. Plasmid DNA was prepared using a GFX plasmid kit (Amersham Biosciences, Freiburg, Germany) and cycle sequencing was performed with a CycleReader Auto kit (MBI Fermentas). Sequences were run and evaluated on an A.L.F.express sequencer (Amersham Biosciences). Long Ranger (Biozym, Hess. Oldendorf, Germany) and ReproGel (Amersham Biosciences), respectively, mixes were used for gel matrix preparation and both gels were subjected to UV light for a uniform polymerization. Additional clones were custom sequenced by Seqlab (Göttingen, Germany). The sequences were submitted to GenBank and assigned accession numbers AY095311 and AY095312.

\section{Sequence and Phylogenetic Analyses}

Sequences X14908 (including variant sites for CSN3 A and B), AF041482, AF123250, AF123251, AF105260, $\mathrm{AF} 121023, \mathrm{AF} 092513, \mathrm{AJ} 619772$, and AF194989 representing $C S N 3$ alleles $A, B, E, F, G, H, I, A^{I}$, respectively, an unassigned Bos taurus sequence (AJ619772), and a yak sequence (AFA194989) were retrieved from GenBank. Based on the CSN3*B sequence, DNA sequences for $C S N 3{ }^{*} C$ and $C S N 3{ }^{*} J$ were deduced from the protein sequences, differing in codons 97 ( $\mathrm{Arg} \rightarrow \mathrm{His}$; Miranda et al., 1993) and 155 (Ser $\rightarrow$ Arg; Mahé et al., 1999), which makes single $\mathrm{G} \rightarrow \mathrm{A}$ and $\mathrm{C} \rightarrow \mathrm{T}$ substitutions most probable. A recognition site search for Hin$d \mathrm{III}$, HinfI, MspI, PstI, and TaqI was done for X14908 (A and B) and the yak sequences AF194989, AY095311, and AY095312 using the pDRAW32 1.0 DNA analysis freeware (AcaClone, 2007). Because of different lengths of the sequences, only partial sequences corresponding to the coding region of exon IV were used for phylogenetic analysis. The Gendoc software (Nicholas and Nicholas, 1997) was used for sequence alignments and cutting. Construction of a DNA nucleotide sequencebased neighbor joining tree using genetic distance $\mathrm{P}$ was done with the DAMBE software package (Xia and Xie, 2001). Randomized input order and bootstrapping with 100 data sets were used to obtain a consensus tree. TreeviewX (Page, 2005) was used to redraw the 


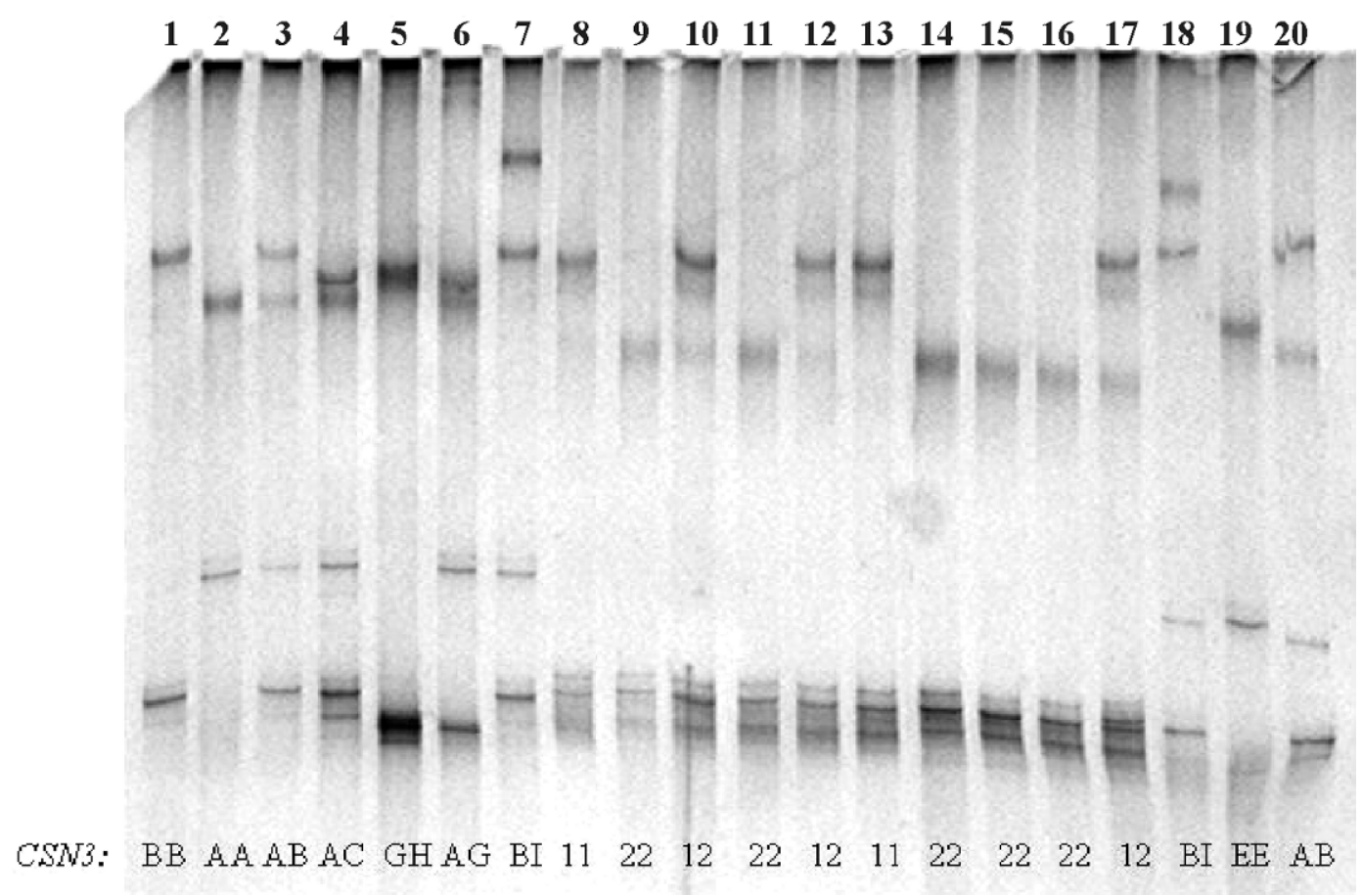

Figure 1. Polymerase chain reaction-single strand conformation polymorphism analysis of the yak CSN3 exon IV. Lanes are numbered above the gel; the genotypes are given below each sample lane. Lanes 1 to 7 and 18 to 20 represent cattle samples with known genotypes, lanes 8 to 17 show yak samples with different migration patterns named 1 (slow migration) and 2 (fast migration) and all possible genotypes 11,12 , and 22 .

phylogram provided in DAMBE as a rectangular cladogram.

\section{RESULTS AND DISCUSSION}

Primers derived from the bovine CSN3 sequence amplified a fragment in the expected size (about $550 \mathrm{bp}$ in agarose gels) in all yak. Analysis by PCR-SSCP revealed 2 distinct migration patterns in the yak samples that did not correspond to any of the known cattle CSN3 variants. The 2 migration patterns were preliminarily named type 1 and type 2 in the order of increasing mobility (Figure 1). The lower fragment of both yak variants moved close to the cattle $\operatorname{CSN}^{*} B$ fragment. Migration of the upper fragments was more different, with positions similar to $B$ (type 1 ) or below the cattle $C S N 3{ }^{*} A$ (type 2, Figure 1). Frequencies of the 2 yak CSN3 PCR-SSCP patterns were 0.32 (type 1) and 0.68 (type 2).

In PCR-RFLP analysis with HindIII, MspI, and TaqI, there was no clear difference between the 2 PCR-SSCP genotypes in the restriction fragments in the agarose gels. All yak samples showed identical restriction fragments. All PCR products were uncut with TaqI and $M s p I$, which indicates $\operatorname{Thr}_{136}$ (corresponding to $C S N 3{ }^{*} A$ in cattle) and excludes the silent mutation in the codon
$\mathrm{Pro}_{150}$ present in $C S N 3 * A^{I}$. With HindIII, all PCR products were cut (about 450 and $100 \mathrm{bp}$ ), as would be expected for $C S N 3^{*} B$ in cattle with Ala ${ }_{148}$. Gel pictures are available in an online-only data supplement (http:// jds.fass.org/content/vol91/issue4/).

A 12-bp sequence duplication in the coding frame of exon IV in PCR-SSCP type 2 (AY095311) was detected by sequencing. Due to the sequence structure, the position of the inserted motif could not be unequivocally assigned. The duplication corresponded to the codons for AA either from 147 to 150 (Glu-Ala-Ser-Pro) or from 148 to 151 (Ala-Ser-Pro-Glu), which were repeated identically. This has led to a total of $164 \mathrm{AA}$ encoded in the yak type 2 CSN3 exon IV, whereas the sequence of yak type 1 (AY095312) had the same length as in cattle, encoding 160 of the $169 \mathrm{AA}$ of mature $\kappa$-casein. Another difference was noticed in the stop codon, which was TAA for type 2, corresponding to all previously known cattle variants, and TGA for type 1 . The deduced AA sequences showed identity with bovine $C S N 3{ }^{*} A$ in position 136 (Thr) and with bovine $C S N 3^{*} B$ in position 148 (Ala), which is in accordance with the results from PCRRFLP analysis. Comparison of AY095311 and AY095312 with yak sequence AF194989 in GenBank showed identical length of the coding region in exon IV and a shared stop codon (TGA) between AY095312 (type 


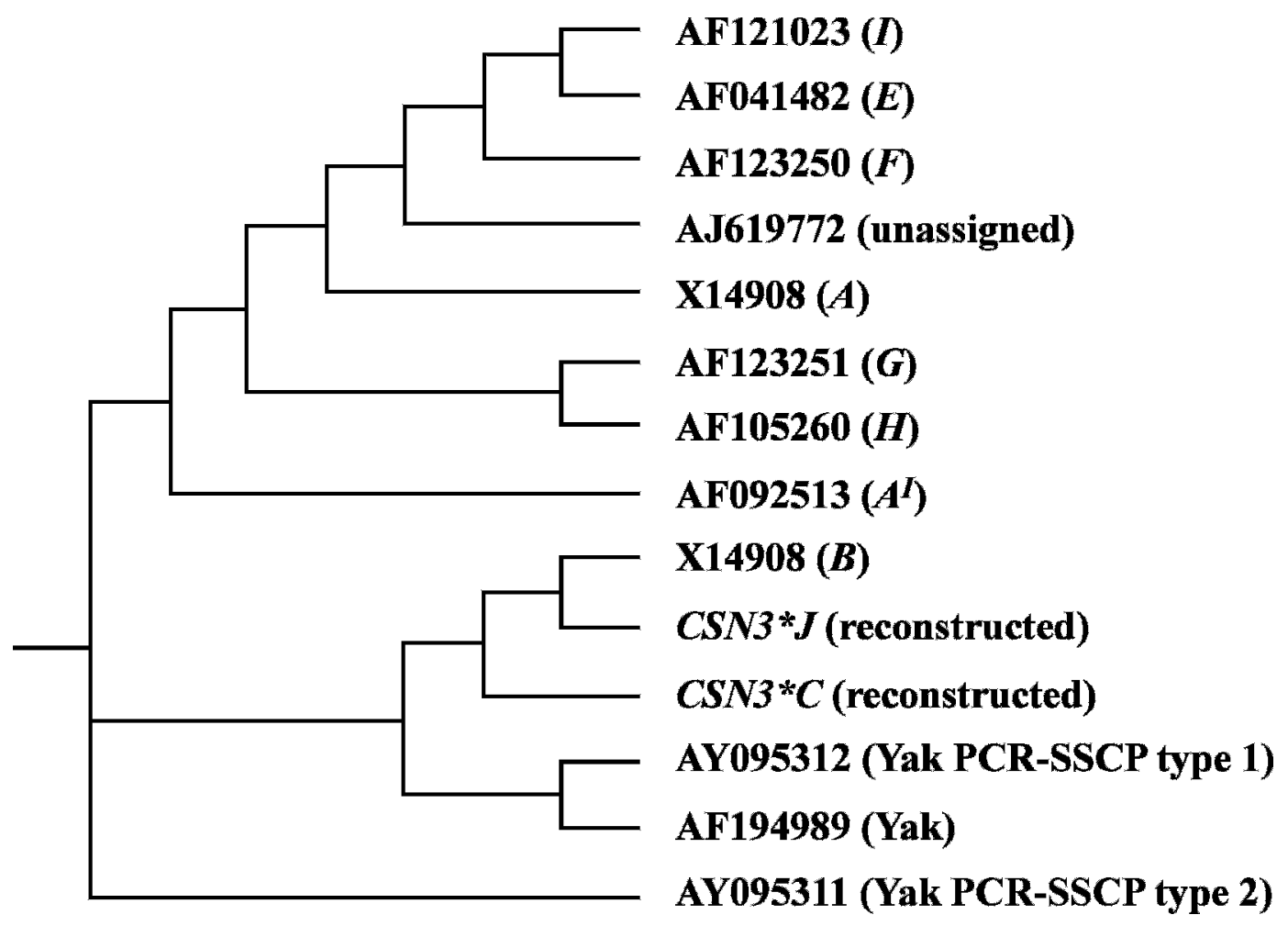

Figure 2. Nucleotide sequence-based neighbor joining consensus tree (random input order, 100 bootstrap datasets) of CSN3 sequences in the genus Bos (Bos taurus, Bos indicus, and Bos grunniens) drawn as ladderized rectangular cladogram. AY095311 was used as outgroup. DNA sequences for $C S N 3 * C$ and $J$ are not available in GenBank and were reconstructed based on protein sequence data assuming single nucleotide mutations.

1) and the sequence AF194989, but 2 additional mutations $(\mathrm{C} / \mathrm{T}$ and $\mathrm{C} / \mathrm{G})$, both changing the AA sequence (codon 36 Pro/Leu and 130 Pro/Arg) were present in AF194989. The in silico restriction analysis using pDRAW32 confirmed the results obtained in PCRRFLP analysis. The restriction sites for HindIII, HinfI, $P s t \mathrm{I}$, and TaqI are indicated in an online-only data supplement (http://jds.fass.org/content/vol91/issue4/). With HindIII, the inserted motif led to a second recognition site in AY095311 and consequently 12 bp were cut out. This makes the insertion undetectable in agarose gel electrophoresis. Although 2 mutations are present in AF194989, pDRAW restriction analysis revealed identical cutting positions in all 3 yak sequences and bovine $C S N 3{ }^{*} B$ for HinfI and PstI. Due to the insertion a 12-bp length difference in the largest fragment resulted in AY095311 compared with the other yak sequences with these 2 enzymes, but this will be hard to discriminate in agarose gel electrophoresis. For TaqI in all 3 yak sequences no restriction site was present, which corresponds to bovine $C S N 3 * A$. Therefore, discrimination of yak CSN3 from the bovine CSN3 variants by PCR-RFLP requires at least 2 enzymes including TaqI and one chosen of the group HindIII, HinfI, and PstI. Reliable discrimination of the yak variants AY095311 and AY095312 by HinfI and PstI furthermore will require high-resolution agarose or even polyacrylamide gel electrophoresis to make the 12-bp difference clearly visible. HindIII, although the most popular enzyme in CSN3 PCR-RFLP analysis, seems the least suitable choice for yak CSN3 variant discrimination, because gel electrophoresis in this case must be able to detect a 12-bp fragment aside from possible excess primers.

Because AY095311 seemed to be the most divergent sequence due to the duplication, AY095311 was used as outgroup in the construction of a neighbor joining tree with DAMBE. The resulting consensus tree (Figure 2 ) shows 3 main branches, 1 with the out-grouped yak sequence and 2 main sequence groups. The nucleotide and AA sequence differences are summarized in Table 1 and provided in full in an online-only data supplement (http://jds.fass.org/content/vol91/issue4/). The first group comprises $C S N 3^{*} A, E, I, F, G, H, A^{I}$, and the unassigned taurine sequence AJ619772, all being identical to $C S N 3 * A$ in codons 136 and 148 . The nucleotide sequences of $C S N 3^{*} E, I, F, H, A^{I}$, and AJ619772 each differ from $C S N 3 * A$ in a single mutation, and thus 
Table 1. Differences in AA and nucleotide sequences of CSN3 variants in the genus Bos $^{1}$

\begin{tabular}{|c|c|c|c|c|c|c|c|c|c|c|c|}
\hline \multirow{2}{*}{$\begin{array}{l}\text { GenBank } \\
\text { no. and } \\
\text { allele name }\end{array}$} & \multicolumn{11}{|c|}{ AA position of mature $\kappa$-casein } \\
\hline & 10 & 36 & 97 & 104 & 130 & 135 & 136 & 148 & $148 \mathrm{a}-151 \mathrm{a}$ & 155 & Stop \\
\hline X14980 & Arg & Pro & Arg & Ser & Pro & Thr & Thr & Asp & & Ser & \\
\hline$A$ & CGC & $\mathrm{CCT}$ & CGT & TCA & CCT & $\mathrm{ACC}$ & ACC & GAT & & AGC & TAA \\
\hline X14908 & & & & & & & Ile & Ala & & & \\
\hline$B$ & & & & & & & ATC & GCT & & & \\
\hline Not available & & & His & & & & Ile & Ala & & & \\
\hline$C$ & & & CAT & & & & ATC & GCT & & & \\
\hline $\begin{array}{l}\text { AF041482 } \\
E\end{array}$ & & & & & & & & & & & \\
\hline $\mathrm{AF} 12350$ & His & & & & & & & & & & \\
\hline$F$ & $\mathrm{CAC}$ & & & & & & & & & & \\
\hline AF123251 & & & Cys & & & Ile & & & & & \\
\hline$G$ & & & TGT & & & ATC & & & & & \\
\hline AF105260 & & & & & & Ile & & & & & \\
\hline$H$ & & & & & & ATC & & & & & \\
\hline AF121023 & & & & Ala & & & & & & & \\
\hline I & & & & GCA & & & & & & & \\
\hline Not available & & & & & & & Ile & Ala & & Arg & \\
\hline$J$ & & & & & & & ATC & GCT & & AGA & \\
\hline AF092513 & & & & & & & & & & & \\
\hline$A^{I}$ & & & & & & & & & & & \\
\hline AJ619772 & & & His & & & & & & & & \\
\hline Unassigned & & & CAT & & & & & & & & \\
\hline AY095311 & & & & & & & & Ala & Ala Ser Pro Glu & & \\
\hline Yak type 2 & & & & & & & & GCT & GCTTCTCCAGAA & & \\
\hline AY095312 & & & & & & & & Ala & & & \\
\hline Yak type 1 & & & & & & & & GCT & & & TGA \\
\hline AF194989 & & Leu & & & Arg & & & Ala & & & \\
\hline Yak & & CTT & & & CGT & & & GCT & & & TGA \\
\hline
\end{tabular}

${ }^{1}$ Only positions deviating from X14980 $(C S N 3 * A)$ are shown. Amino acids are provided in the upper lane, and nucleotide codons in the lower lane of each sequence. Position of the duplication is indicated as 148a$151 \mathrm{a}$, although $147 \mathrm{a}-150 \mathrm{a}$ is also possible.

${ }^{2} \kappa$-Casein allele name.

probably directly evolved from $C S N 3 *$ A. Allele $C S N 3 * G$ differs from $C S N 3 * A$ in 2 positions, 1 identical to $C S N 3 * H$. Thus, $C S N 3^{*} G$ probably evolved from $C S N 3{ }^{*} H$ by a single mutation. The second main branch is subdivided into the 3 bovine sequences corresponding to $C S N 3{ }^{*} B, C$, and $J$ (group "B", with $C S N 3{ }^{*} C$ and $J$ developing from $C S N 3{ }^{*} B$ by single mutations) and another branch with the 2 yak sequences AY095312 and AF194989, which both differ from all other sequences by the unique TGA stop codon. Because of this stop codon, the sequence AY0095312 is most probably not a common ancestor of $C S N 3^{*} A$ and $C S N 3^{*} B$, although the deduced protein sequence is exactly the intermediate sequence regarding AA 136 and 148. It is unlikely, however, that an identical mutation in the stop codon has occurred in both branches " $\mathrm{A}$ " and "B" after the sequence divergence. The ideal common ancestor of today's $C S N 3 * A$ and $C S N 3 * B$ as postulated by Mercier et al. (1976) would correspond to AY095311 without the 12-bp duplication. Such a sequence was not found in samples from the current study, which might be an effect of the low sample number. The sequence originally reported in bison (Udina et al., 1995) might represent such an ancestral sequence, although data on the stop codon are not reported. As the Bos and Bison genera diverged before Bos taurus/indicus and Bos grunniens departed (Ritz et al., 2000), the same ancestral protein might exist in Bison and eventually also in Bos.

The sequence AY095312, corresponding to PCRSSCP type 1 in this study, might be identical to the report of Sulimova et al. (1996), including the mutated stop codon in yak. The sequence AY095311, including the 12-bp duplication, is most probably new at the molecular level. However, it cannot be excluded that the resulting protein of AY095311, AY095312, or AF194989 may be identical to the variant " $\mathrm{X}$ " described in the early 1990s in yak (Kawamoto et al., 1992).

Two genetic variants of yak CSN3 were detected by PCR-SSCP analysis simultaneously with bovine CSN3 variants and show clearly different migration patterns. This technique enables reliable genotyping of yak CSN3 variants. Analysis by PCR-RFLP using HindIII and TaqI and agarose gels for fragment separation distinguishes yak and bovine variants, but does not discriminate between the 2 yak variants. 


\section{CONCLUSIONS}

$\kappa$-Casein was found to be polymorphic in domesticated yak at the DNA sequence level. The deduced protein sequences were also different, but in the current study no milk samples were available to check electrophoretic mobility. At least 2 yak-specific sequences are present and can be discriminated by PCR-SSCP or sequence analysis. This can be considered in future studies on the genetic variation within and between yak populations, on the introgression of cattle genes into domesticated yak, and on the influence of CSN3 polymorphisms on technological or nutritional parameters of yak milk and association with production traits.

\section{ACKNOWLEDGMENTS}

The authors thank H. Wagner, M. Uebel, and T. Gans for assistance in cloning and sequencing. H. Jianlin was supported by a grant of the Yak and Camel Foundation in Germany.

\section{REFERENCES}

AcaClone. 2007. pDRAW32 DNA analysis software. 1.0, Revision 1.1.97 ed. AcaClone Software. www.acaclone.com

Alexander, L. J., A. F. Stewart, A. G. Mackinlay, T. V. Kapelinskaya, T. M. Tkach, and S. I. Gorodetsky. 1988. Isolation and characterization of the bovine kappa-casein gene. Eur. J. Biochem. 178:395-401.

Bassam, B. J., G. Caetano-Anollés, and P. M. Gresshoff. 1991. Fast and sensitive silver staining of DNA in polyacrylamide gels. Anal. Biochem. 196:80-83.

Fan, B. L., N. Li, and C. X. Wu. 2000. Research on constructing phylogenetic trees of ruminants basing on the database of milk protein gene sequences. Chin. J. Genet. 27:485-497. [in Chinese with English abstract]

Farrell, H. M., Jr., R. Jimenez-Flores, G. T. Bleck, E. M. Brown, J. E. Butler, L. K. Creamer, C. L. Hicks, C. M. Hollar, K. F. NgKwai-Hang, and H. E. Swaisgood. 2004. Nomenclature of the proteins of cows' milk-Sixth revision. J. Dairy Sci. 87:1641-1674.

Formaggioni, P., A. Summer, M. Malacarne, and P. Mariani. 1999. Milk protein polymorphism: Detection and diffusion of the genetic variants in Bos genus. Annali della Facoltà di Medicina Veterinaria. Università di Parma 19:127-165.

Gorodetskii, S. I., and A. S. Kaledin. 1987. Nucleotide sequence of the cDNA of kappa casein in cows. Genetika 23:596-604. [Russian with English abstract]
Kawamoto, Y., T. Namikawa, A. Adachi, T. Amano, T. Shotake, T. Nishida, Y. Hayashi, B. Kattel, and H. B. Rajubhadary. 1992. A population genetic study on yaks, cattle and their hybrids in Nepal using milk protein variations. Anim. Sci. J. 63:563-575.

Mahé, M. F., G. Miranda, R. Queval, A. Bado, P. S. Zafindrajaona, and F. Grosclaude. 1999. Genetic polymorphism of milk proteins in African Bos taurus and Bos indicus populations. Characterization of variants as1-Cn H and k-Cn J. Genet. Sel. Evol. 31:239-253.

Mao, Y. J., G. H. Zhong, Y. C. Zheng, X. W. Peng, Z. P. Yang, Y. Wang, and M. F. Jiang. 2004. Genetic polymorphism of milk protein and their relationships with milking performances in Chinese yak. Sci. Agric. Sinica 3:310-315. [Chinese with English abstract]

Mercier, J.-C., J.-M. Chobert, and F. Addeo. 1976. Comparative study of the amino acid sequences of the caseinomacropeptides from seven species. FEBS Lett. 72:208-214.

Miranda, G., P. Anglade, M. F. Mahé, and G. Erhardt. 1993. Biochemical characterization of the bovine genetic kappa-casein $\mathrm{C}$ and $\mathrm{E}$ variants. Anim. Genet. 24:27-31.

Montgomery, G. W., and J. A. Sise. 1991. Extraction of DNA from sheep white blood cells. N. Z. J. Agric. Res. 33:437-441.

Nicholas, K. B., and H. B. Nicholas Jr. 1997. Subject: GeneDoc: A tool for editing and annotating multiple sequence alignments. www.nrbsc.org/gfx/genedoc/index.html Accessed Jan. 25, 2008.

Page, R. D. M. 2005. TreeviewX. 0.5.0 ed. Available online at http:// darwin.zoology.gla.ac.uk/ rpage/treeviewx/

Prinzenberg, E. M., I. Krause, and G. Erhardt. 1999. SSCP analysis at the bovine CSN3 locus discriminates six alleles corresponding to known protein variants $(A, B, C, E, F, G)$ and three new DNA polymorphisms $\left(H, I, A^{1}\right)$. Anim. Biotechnol. 10:49-62.

Ritz, L. R., M. L. Glowatzki-Mullis, D. E. MacHugh, and C. Gaillard. 2000. Phylogenetic analysis of the tribe Bovini using microsatellites. Anim. Genet. 31:178-185.

Sulimova, G. E., Y. N. Badagueva, and I. G. Udina. 1996. Polymorphism of the kappa-casein gene in populations of the subfamily Bovinae. Russ. J. Genet. 32:1371-1386.

Udina, I. G., Y. N. Badagueva, G. E. Sulimova, and I. A. Zakharov. 1995. Distribution of the kappa-casein gene alleles in the bison (Bison bonasus) population. Russ. J. Genet. 31:1452-1453.

Xia, X., and Z. Xie. 2001. DAMBE: Software package for data analysis in molecular biology and evolution. J. Hered. 92:371-373.

Xuebin, Q., and H. Jianlin. 2000. Introgression makes yak populations genetically different: Evidence from beta-lactoglobulin variations. Pages 202-208 in Yak Production in Central Asian Highlands. Proc. 3rd Int. Congr. on Yak, Lhasa, China. http://www. ilri.org/InfoServ/Webpub/Fulldocs/Yakpro/SessionB4.htm Accessed Jan. 25, 2008.

Yucai, Z., Z. Guanghui, M. Yongjiang, P. Xianwen, J. Mingfeng, W. Yong, Z. Sixiang, and C. Jie. 2000. Milk protein genetic polymorphisms: A comparison between Maiwa yak and Jiulong yak. Pages 209-212 in in Yak Production in Central Asian Highlands. Proc. 3rd Int. Congr. on Yak, Lhasa, China. http://www.ilri.org/Info Serv/Webpub/Fulldocs/Yakpro/SessionB5.htm Accessed Jan. $25,2008$. 